\title{
Comparison of the Gut Microbiota of Centenarians in Longevity Villages of South Korea with Those of Other Age Groups ${ }^{\text {s }}$
}

\author{
Bong-Soo Kim ${ }^{1,2+}$, Chong Won Choi ${ }^{3,4 \dagger}$, Hyoseung Shin ${ }^{3,4}$, Seon-Pil Jin ${ }^{3,4,5}$, Jung-Soo Bae ${ }^{3,4,5}$, Mira Han ${ }^{3,4,5}$, \\ Eun Young Seo ${ }^{3,4}$, Jongsik Chun ${ }^{6}$, and Jin Ho Chung ${ }^{3,4,5,7 *}$ \\ ${ }^{1}$ Department of Life Science, Hallym University, Chuncheon 24252, Republic of Korea \\ ${ }^{2}$ Multidisciplinary Genome Institute, Hallym University, Chuncheon 24252, Republic of Korea \\ ${ }^{3}$ Department of Dermatology, Seoul National University College of Medicine, Seoul 03080, Republic of Korea. \\ ${ }^{4}$ Laboratory of Cutaneous Aging Research, Biomedical Research Institute, Seoul National University Hospital, Seoul 03080, Republic of Korea \\ ${ }^{5}$ Department of Biomedical Science, Seoul National University Graduate School, Seoul 03080, Republic of Korea \\ ${ }^{6}$ School of Biological Sciences and Inst. of Molecular Biology and Genetics, Seoul National University, Seoul 08826, Republic of Korea \\ ${ }^{7}$ Institute on Aging, Seoul National University, Seoul 03080, Republic of Korea
}

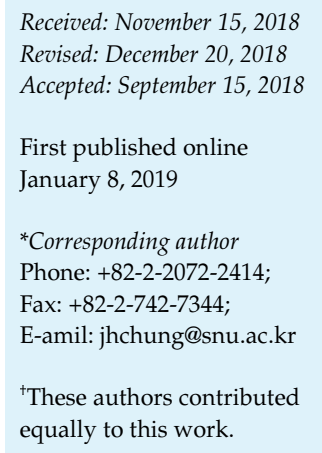

S upplementary data for this paper are available on-line only at http://jmb.or.kr.

pISSN 1017-7825, eISSN 1738-8872

Copyright(C 2019 by

The Korean Society for Microbiology and Biotechnology

\begin{abstract}
Several studies have attempted to identify factors associated with longevity and maintenance of health in centenarians. In this study, we analyzed and compared the gut microbiota of centenarians in longevity villages with the elderly and adults in the same region and urbanized towns. Fecal samples were collected from centenarians, elderly, and young adults in longevity villages, and the gut microbiota sequences of elderly and young adults in urbanized towns of Korea were obtained from public databases. The relative abundance of Firmicutes was found to be considerably higher in subjects from longevity villages than those from urbanized towns, whereas Bacteroidetes was lower. Age-related rearrangement of gut microbiota was observed in centenarians, such as reduced proportions of Faecalibacterium and Prevotella, and increased proportion of Escherichia, along with higher abundances of Akkermansia, Clostridium, Collinsella, and uncultured Christensenellaceae. Gut microbiota of centenarians in rehabilitation hospitals were also different to those residing at home. These differences could be due to differences in diet patterns and living environments. In addition, phosphatidylinositol signaling system, glycosphingolipid biosynthesis, and various types of $\mathrm{N}$-glycan biosynthesis were predicted to be higher in the gut microbiota of centenarians (corrected $p<0.05)$. These three metabolic pathways of gut microbiota can be associated with the immune status and healthy gut environment of centenarians. Although further studies are necessary to validate the function of microbiota between groups, this study provides valuable information on centenarians' gut microbiota.
\end{abstract}

Keywords: Centenarian, gut microbiota, longevity village, rehabilitation hospital

\section{Introduction}

Centenarians are individuals of exceptionally advanced age $[1,2]$. These individuals have been reported to present lower incidence of chronic illness, reduced morbidity and an extended healthy lifespan [3-6]. Because of the suppressed mortality and morbidity, centenarians have been regarded as a model for healthy aging and some studies have been performed to find the genetic and/or environmental factors that might play a role in healthy and disease free aging [7]. However, information on longevity factors is limited and further studies are necessary to find such longevity attributes.

Recently, several studies revealed that the human gut microbiome shows differences based on the age, place of residence, and diet of different individuals [8, 9]. Association 
of the human gut microbiome with metabolic disorder, obesity, inflammatory bowel disease, and infections has also been analyzed [10,11]. The possible determination of healthy aging by gut microbiome was proposed due to its effects on human metabolism and immune system [12,13]. Studies reported that with aging there was a reduction of bacterial diversity and increased colonization of opportunistic pathogens, such as Staphylococcaceae, Enterobacteriaceae, and Enterococcaceae, along with a reduction of beneficial microbes such as short-chain fatty acid (SCFA)-producing bacteria [12, 14-18]. The gut microbiota of the elderly has been associated with the host immune homeostasis via inflammation and can contribute to the progression of diseases and frailty in the elderly [19-21]. However, the gut microbiota in centenarians has higher bacterial diversity, which is likely linked to good immunological and metabolic health [18]. Although similar results of gut microbiota with aging were observed in previous studies, there were differences regarding the precise changes of gut microbiota. These discrepancies could be due to the differences in diet, lifestyle, genetic factors, and analysis methods [22]. In addition, gut microbiota analyzed in previous studies ( 8 in China, 7 in Japan, and 39 in Italy) could be insufficient to characterize the gut microbiota in centenarians. Therefore, there is a need to analyze gut microbiota of centenarians and collect the observations in a public database. This information and the potential modulation of gut microbiota can improve the health of the elderly.

The neighboring counties of Gurye, Gokseong, Sunchang, and Damyang, located in the southwestern part of Korea, are reported to form a longevity belt with a high prevalence of centenarians (KOSIS, 2017). Although a previous study has reported the differences in the gut microbiota in these regions compared with those in urbanized towns [16], the gut microbiota of the local centenarians were not analyzed. The gut microbiome might be one of the important factors in the prevalence of centenarians in these regions. Therefore, in this study, we analyzed the gut microbiota of centenarians living in these four regions and compared the results to those of healthy elderly individuals and adults in the same regions and urbanized towns. This study will provide comprehensive information of the gut microbiota of centenarians in longevity villages and help to characterize the role of gut microbiota in healthy aging.

\section{Materials and Methods}

\section{Study Subjects and Sample Collection}

Thirty centenarians (aged 95 to 108 years; average $98.9 \pm 3.4$ ), 17 elderly (aged 67 to 79 years; average $73.6 \pm 3.6$ ), and 9 adults (aged
26 to 43 years; average $34.3 \pm 6.5$ ) in longevity villages were enrolled in this study. Of these, 20 centenarians had lived in the community and 10 had lived in rehabilitation hospitals. Subjects who were affected by malignant neoplasia (stage 3 or more), uncontrolled chronic diseases (such as uncontrolled hypertension or uncontrolled diabetes), active systemic infectious diseases, and alcoholics were excluded from the study. In addition, subjects who had used systemic antibiotics within one month were also excluded. A written consent form and completed questionnaire regarding the age, gender, past medical history, use of antibiotics, and characteristics of diet were obtained from each of the subjects. The study protocol was approved by the Ethical Community of Seoul National University Hospital (IRB No: H-1210-091-435). Fecal samples were collected from enrolled subjects and immediately stored in ice-boxes. Samples were transported in iceboxes to laboratory and stored at $-80^{\circ} \mathrm{C}$ before DNA extraction.

\section{DNA Extraction and Pyrosequencing}

Total DNA was extracted from fecal samples of subjects using a FastDNA SPIN Extraction Kit (MP Biomedicals, USA). Variable regions of V1-V3 in 16S rRNA gene were amplified and used to prepare a library for pyrosequencing using barcoded fusion primers $[23,24]$. In brief, amplification was conducted in a final volume of $50 \mu \mathrm{l}$ with $10 \times$ Taq buffer, dNTP mixture (Takara, Japan), $10 \mu \mathrm{M}$ of each barcoded primer, and $2 \mathrm{U}$ of Taq polymerase (ExTaq; Takara) using a C1000 Touch thermal cycler (Bio-Rad, USA). Amplified products were purified using the QIAquick PCR Purification Kit (Qiagen, USA), and the quantification of products was performed using a PicoGreen dsDNA Assay Kit (Invitrogen, USA). Equimolar concentrations of each product were pooled and purified again using an AMPure Bead Kit (Agencourt Bioscience, USA). Sequencing was conducted on a Roche/454 GS FLX+ system according to the manufacturer's protocols.

\section{Sequence Data Analysis}

To compare the gut microbiota of subjects in longevity villages with healthy adults and the elderly in urbanized towns, sequence data of individuals from urbanized towns in previous studies [16, 25] were obtained from public database (SRP052893 in NCBI short read archive and ERP002551 in EMBL SRA database). Downloaded sequences with low read number $(<3,300$ reads after quality filtering), and ambiguous age information (aged 20 to 48 years for adults; aged 60 to 75 years for the elderly) were excluded in further analyses. All obtained sequences from public database and present study were generated from the same pyrosequencing system. Sequences obtained from present study $(n=56)$ and public database $(n=26)$ were analyzed using the CLC genomic workbench (ver. 8.5.1) with the Microbial Genomics Module (Qiagen). Sequences were separated by unique barcodes, and lowquality reads (average quality score $<25$ or read length $<300 \mathrm{bp}$ ) and primer sequences were removed using USEARCH program [26]. Chimeric sequences were removed using the UPARSE tool [27]. Sequences were clustered into operational taxonomic units 
(OTUs) at $97 \%$ similarity, and representative sequences in each cluster were identified using RDP classifier [28] with the EzTaxon-e database [29]. The read numbers in each sample were normalized by random sampling, and the diversity indices were calculated using the Mothur program [30]. Principal coordinate analysis (PCoA) was performed to compare the microbiota among groups, based on the Bray-Curtis distance using Calypso [31]. Heatmap analyses and random forest test were performed using $\mathrm{R}$ software (ver. 3.2.2). The LEfSe (linear discriminant analysis coupled with effect size measurements) was used to find potential markers among different groups [32]. The functional prediction of microbiota was performed using the PICRUSt (ver. 1.0.0) [33]. All raw sequence reads obtained in this study are available in the EMBL SRA database under study number PRJEB7507 (http:// www.ebi.ac.uk/ena/data/view/PRJEB7507).

\section{Statistical Analysis}

The differences of bacterial taxa among groups were tested by Kruskal-Wallis rank-sum test in R software. Mann-Whitney U-test was performed to identify statistically significant pairwise differences between the groups. Significantly different predicted KEGG pathways were determined using the Kruskal-Wallis Htest, and a post-hoc test was performed using the Tukey-Kramer method [34]. The multiple test corrections were made using Benjamini-Hochberg False Discovery Rate. Results with $p$ value $<0.05$ were considered statistically significant.

\section{Results}

\section{Characteristics of the Enrolled Subjects}

The characteristics of the enrolled subjects in this study are summarized in Table S1. Centenarian subjects were
$90 \%$ female and $10 \%$ male, whereas the percentages of female among the elderly and adults were $41.2 \%$ and $33.3 \%$, respectively. All of the elderly and adults were community-dwelling, while ten subjects among the centenarians resided in a rehabilitation hospital and the others were community-dwelling (at home). The mean age of centenarians residing at home and rehabilitation hospital was $99.1 \pm 3.5$ and $98.7 \pm 3.6$, respectively.

The dietary data of the subjects were collected through a food frequency questionnaire including the status of appetite, the numbers of meals and snacks per day, and the consumption frequency of each food group per month (Table 1). The number of meals and snacks was higher among centenarians and the elderly group compared with that of the adult group $(p<0.001$ and $p=0.004)$. The questionnaire about the status of appetite also showed no differences between the groups as $(72.0 \%(18 / 25)$ of centenarians, $53.8 \%(7 / 13)$ of elderly, and $77.8 \%(7 / 9)$ of adults had good appetite, $p=0.411)$. The frequencies of intake of each food group (meat, eggs, fish, bean curd, fermented soybean pastes, dairy products, and fruits) were compared and it was found that adults $(17.7 \pm 13.3 /$ month $)$ consumed eggs more frequently than the centenarians (8.1 $\pm 8.3 /$ month $)$ and the elderly $(6.7 \pm 5.7 /$ month $)(p=0.015)$. Although statistically insignificant, the adults consumed meat more frequently, while the centenarians and the elderly consumed fish and bean curd more frequently. The frequency of intake of protein-containing food (sum of meat, eggs, fish, and bean curd) was not significantly different between the centenarians, elderly, and adults ( $p=$

Table 1. Dietary characteristics of each group.

\begin{tabular}{|c|c|c|c|c|}
\hline & Centenarians & Elderly & Adult & $p$ value \\
\hline Subjects (n) & 25 & 13 & 9 & \\
\hline Number of meals (per day) & $2.84 \pm 0.37$ & $3.00 \pm 0.00$ & $2.33 \pm 0.50$ & $<0.001$ \\
\hline Number of snacks (per day) & $1.06 \pm 0.82$ & $1.69 \pm 0.85$ & $0.50 \pm 0.61$ & 0.004 \\
\hline Frequency of intake (per month) & Centenarians & Elderly & Adult & $p$ value \\
\hline Subjects (n) & 22 & 12 & 9 & \\
\hline Meat & $13.5 \pm 22.7$ & $10.8 \pm 12.1$ & $16.4 \pm 13.0$ & 0.787 \\
\hline Eggs & $8.1 \pm 8.3$ & $6.7 \pm 5.7$ & $17.7 \pm 13.3$ & 0.015 \\
\hline Fish & $8.8 \pm 9.7$ & $8.6 \pm 10.6$ & $5.0 \pm 2.5$ & 0.536 \\
\hline Bean curd & $12.7 \pm 12.9$ & $19.1 \pm 25.0$ & $9.0 \pm 5.8$ & 0.354 \\
\hline Fermented soybean pastes & $28.6 \pm 27.1$ & $35.8 \pm 28.2$ & $9.8 \pm 5.4$ & 0.061 \\
\hline Fruits & $12.7 \pm 13.2$ & $20.4 \pm 20.2$ & $21.7 \pm 14.5$ & 0.237 \\
\hline Eat protein & $43.1 \pm 37.3$ & $45.1 \pm 25.8$ & $48.1 \pm 26.7$ & 0.925 \\
\hline
\end{tabular}


A
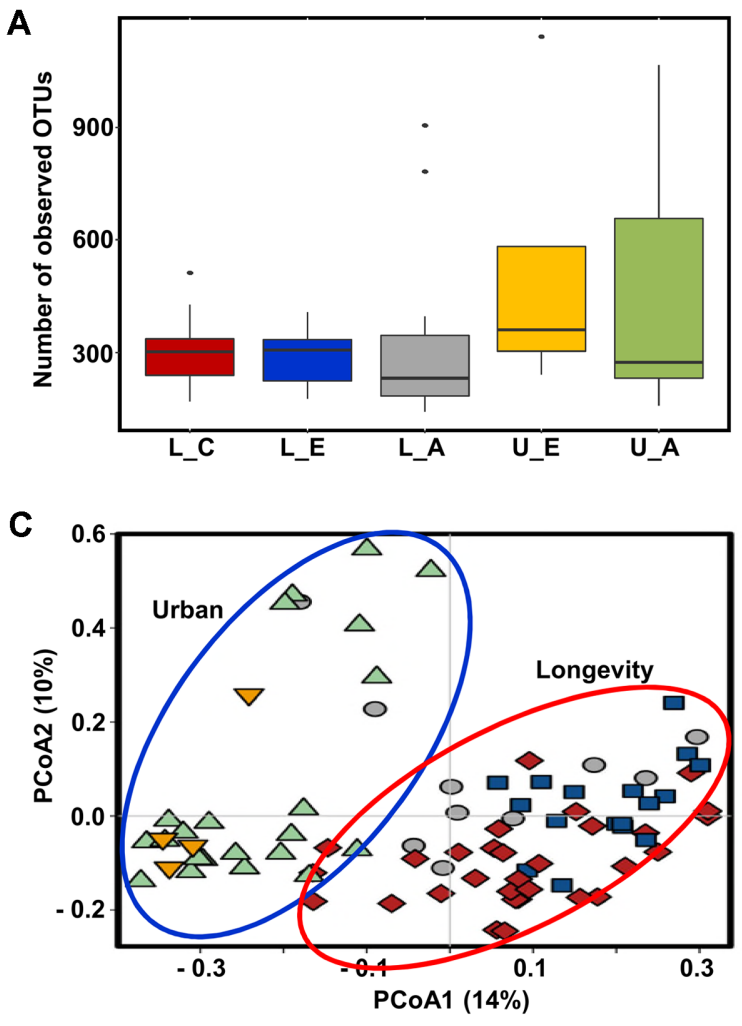

$>$ L_C L_E $O$ L_A $\nabla$ U_E $\triangle$ U_A
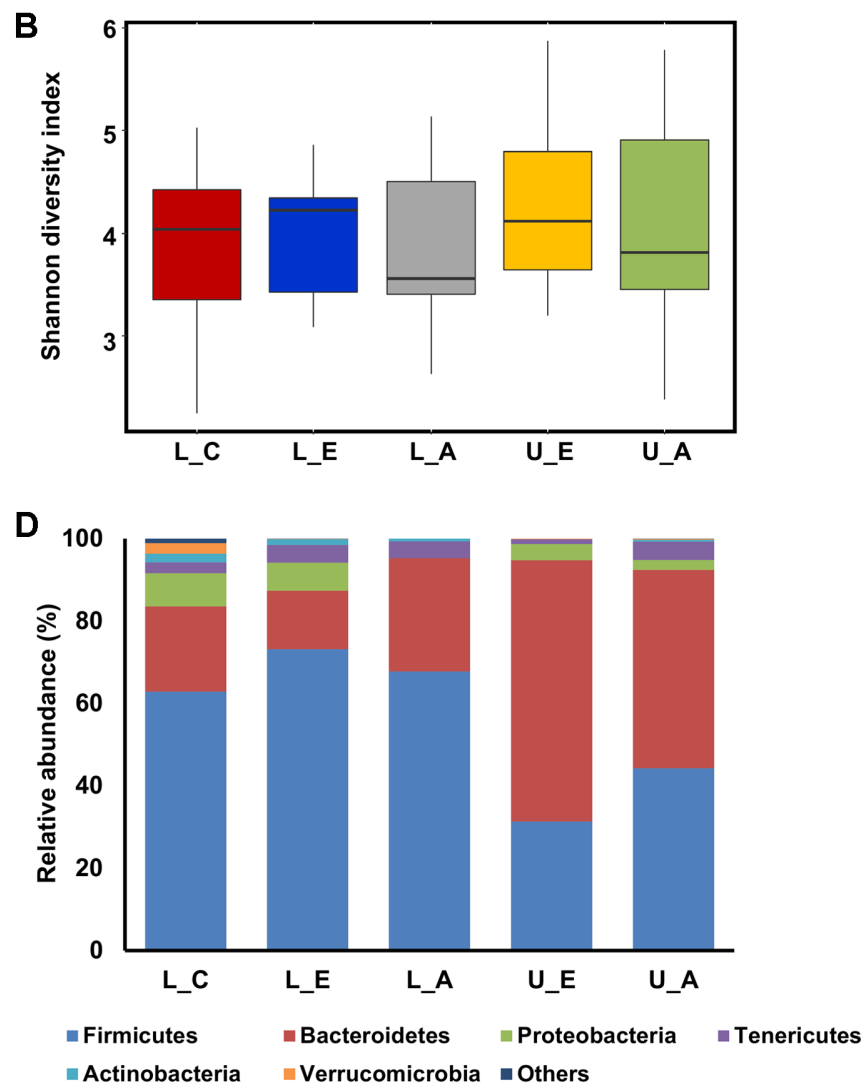

Fig. 1. Comparison of the gut microbiota among groups.

(A) Comparison of observed OTUs between centenarians, elderly, and adults in longevity villages and urbanized towns. (B) Comparison of Shannon diversity indices among the groups. (C) The gut microbiota among groups was compared using the PCoA plot based on Bray-Curtis distance. (D) Phylum composition was compared among groups. L_C, centenarians in longevity villages; L_E, elderly in longevity villages; L_A, adults in longevity villages; U_E, elderly in urbanized town; U_A, adults in urbanized town.

0.925). In addition, the frequency of intake of fermented soybean pastes by the centenarians $(28.6 \pm 27.1 /$ month $)$ and the elderly $(35.8 \pm 28.2 /$ month $)$ was higher than that by the adults $(9.8 \pm 5.4 /$ month $)(p=0.061)$.

\section{Comparison of Gut Microbiota between Subjects in Longevity Villages and Urbanized Towns}

Sequences obtained from this study (30 centenarians, 17 elderly, and 9 adults) and public database (2 adults in longevity village, 22 adults in urbanized town, and 4 elderly in urbanized town were used after quality filtering) were compared using the same sequence analysis pipeline. Although the observed OTUs and Shannon diversity indices were higher in subjects of urbanized towns than in longevity villages, these differences were not statistically significant $(p>0.05)$ (Fig. 1). The gut microbiota of subjects in longevity villages were separated from subjects in urbanized towns in PCoA plot (Fig. 1C). The relative abundances of Firmicutes were higher in subjects of longevity villages than in urbanized towns $(p<0.05)$, whereas the proportions of Bacteroidetes were higher in urbanized towns (Fig. 1D). The significance of phyla pairwise difference between groups is presented in Table S2.

The difference of gut microbiota between subjects in longevity villages and urbanized towns was also observed in heatmap analysis based on the proportion of selected taxa (Fig. S1). The compared taxa were selected from consistent results from LEfSe analysis and random forest analysis between subjects in longevity villages and urbanized towns. The relative abundances of taxa in cluster I were higher in subjects of urbanized towns, whereas those in cluster II were higher in subjects in longevity villages. Frequently detected genera (greater than $0.5 \%$ of average proportions in all subjects) were compared among groups. Twelve genera were significantly different between subjects in longevity villages and 
urbanized towns (Fig. S2). The relative abundances of Intestinibacter, Romboutsia, Turicibacter, and Eubacterium_g5 were higher in subjects of longevity villages than in subjects from urbanized towns $(p<0.05)$. The proportion of Ruminococcus_g2 was higher in the elderly in longevity villages than adults in longevity villages and urbanized towns $(p<0.05)$. Lactobacillus was more abundant in subjects of longevity villages than the elderly and adults in urbanized towns $(p<0.05)$. The proportion of Dialister was lower in centenarians than adults in urbanized towns $(p<0.05)$, whereas Blautia were higher in subjects of longevity villages than adults in urbanized towns $(p<0.01)$. In contrast, the proportions of Lachnospira, uncultured Lachnospiraceae, Roseburia, and Bacteroides were higher in subjects of urbanized towns than longevity villages $(p<0.05)$.

\section{Comparison of Gut Microbiota among Adults, Elderly, and Centenarians in Longevity Villages}

Diversity indices of gut microbiota obtained in this study after normalized reads are presented in Table S2. A total of 355,462 sequences were analyzed from the 56 fecal samples after filtering low quality reads $(202,613$ sequences from centenarians, 102,022 sequences from the elderly, and 50,827 sequences from adults). The highest number of OTUs (512) was detected in the centenarian sample of C28 and the lowest number (143) was detected in the adult sample of A4. The median values of observed OTUs and Shannon diversity indices in samples of centenarians and the elderly were higher than that of adults (Figs. 1A and 1B). However, these differences were not statistically significant $(p>0.05)$.

Two phyla, Firmicutes and Bacteroidetes predominated, followed by Proteobacteria, Tenericutes, and Actinobacteria in the gut microbiota of longevity villages (Fig. 1C). The average phyla composition of centenarians was different from those of the elderly and adults in the same region (significances of difference in Table S2). More diverse phyla were detected in the microbiota of centenarians compared to the elderly and adults. The relative abundance of Verrucomicrobia was significantly higher in centenarians $(2.52 \%)$ than the elderly $(0.12 \% ; p<0.05)$. The relative abundances of Proteobacteria $(8.05 \%)$, Actinobacteria $(2.17 \%)$, and Verrucomicrobia $(2.52 \%)$ were significantly higher in centenarians than those of adults $(0.06 \%$ with $p<0.001,0.61 \%$ with $p<0.05$, and $0.00 \%$ with $p<0.01$, respectively). The compositions of phyla from the elderly were also different from those of adults. The proportion of Proteobacteria was higher in the elderly $(6.47 \%)$ than in adults $(p<0.05)$, whereas Bacteroidetes was lower in the elderly $(14.25 \%)$ than in adults $(27.50 \% ; p<0.05)$.
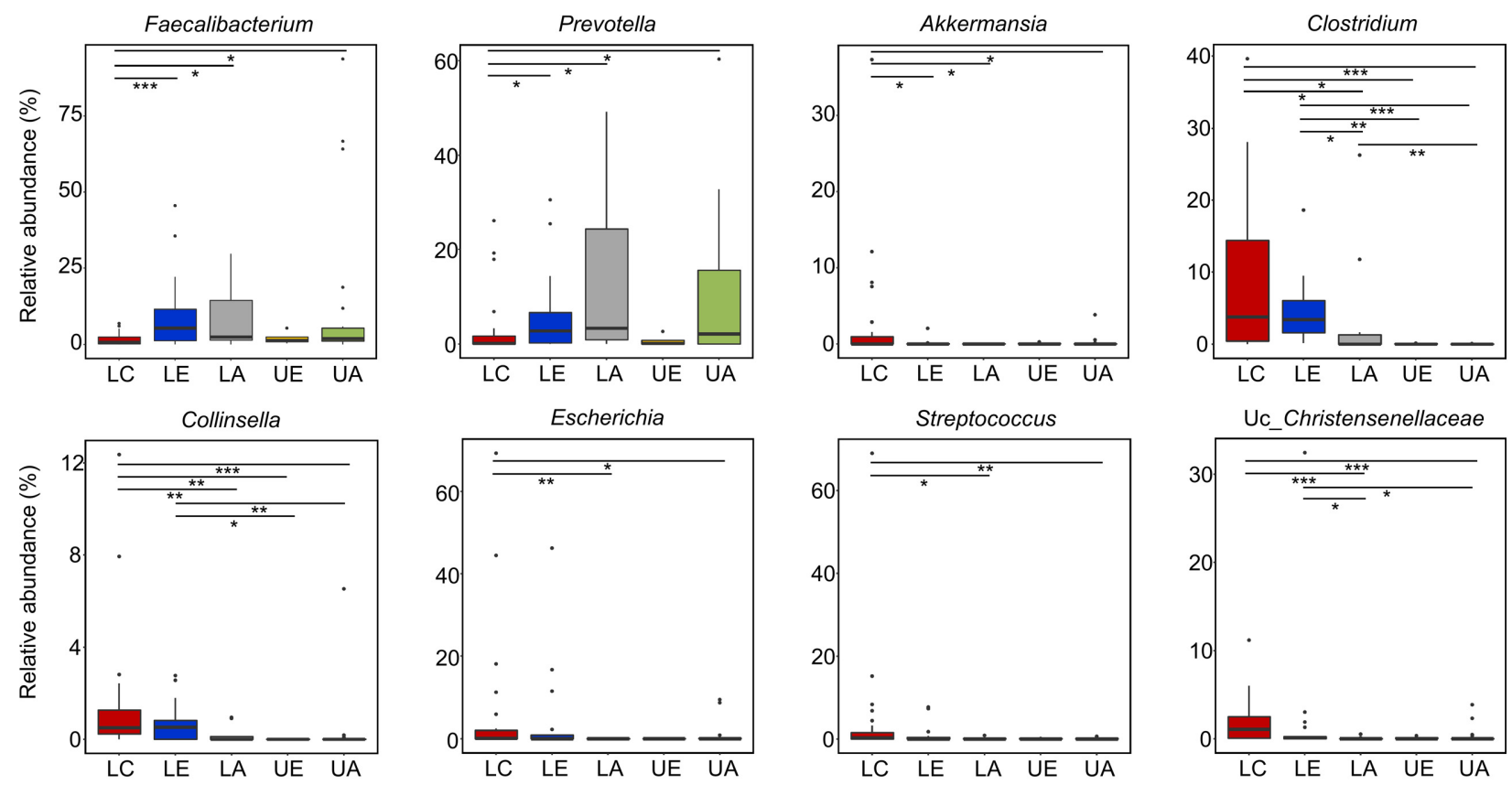

Fig. 2. Comparison of most frequently detected genera among groups.

Genera that comprised more than $0.5 \%$ of all subjects were selected and compared. The significance of the differences between groups was tested using the Mann-Whitney test. L_C, centenarians in longevity villages; L_E, elderly in longevity villages; L_A, adults in longevity villages; U_E, elderly in urbanized town; U_A, adults in urbanized town. $\left({ }^{* * *} p<0.001 ;{ }^{* *} p<0.01 ;{ }^{*} p<0.05\right)$. 
The difference in the bacterial composition among centenarians, the elderly, and adults were detailed at genus level (Fig. 2). Frequently detected genera were compared among groups. The relative abundances of Akkermansia, Clostridium, Collinsella, Escherichia, Streptococcus, and uncultured Christensenellaceae were higher in centenarians than the elderly and adults in longevity villages $(p<0.05)$. In contrast, the proportions of Faecalibacterium and Prevotella were lower in centenarians than the elderly and adults $(p<0.05)$. The decreased proportions of Faecalibacterium and Prevotella were also detected in subjects of urbanized towns according to aging. The heatmap analysis of genera was used to compare the gut microbiota among centenarians, the elderly, and adults in longevity villages using the Spearman rank correlation (Fig. 3). Although the microbiota of centenarians, the elderly, and adults were not separated clearly, microbiota of centenarians in rehabilitation hospitals were clustered together, apart from those of communitydwelling centenarians.
Predicted Functions of Gut Microbiome from Adults, Elderly, and Centenarians in Longevity Villages

The function of gut microbiota was predicted by the PICRUSt using KEEG pathway categories. The relative abundances of the highest KEGG categories were compared among groups in longevity villages (Fig. 4A). The proportion of genes related to metabolism was higher in gut microbiota of centenarians and adults than that in the elderly $(p<0.05)$. Genetic information processing of gut microbiome was reduced by aging $(p<0.05)$. The proportion of environmental information processing was higher in the gut microbiomes of centenarians and the elderly than that in adults. The significantly different metabolic pathway was selected using Kruskal-Wallis H-test with BenjaminiHochberg correction (Table S4). A total of 26 categories were selected by corrected $p$ value $<0.05$. Most of the selected metabolic pathways were found to alter according to aging. Three metabolic pathways among the selected 26 categories were predicted centenarian-specific pathways,

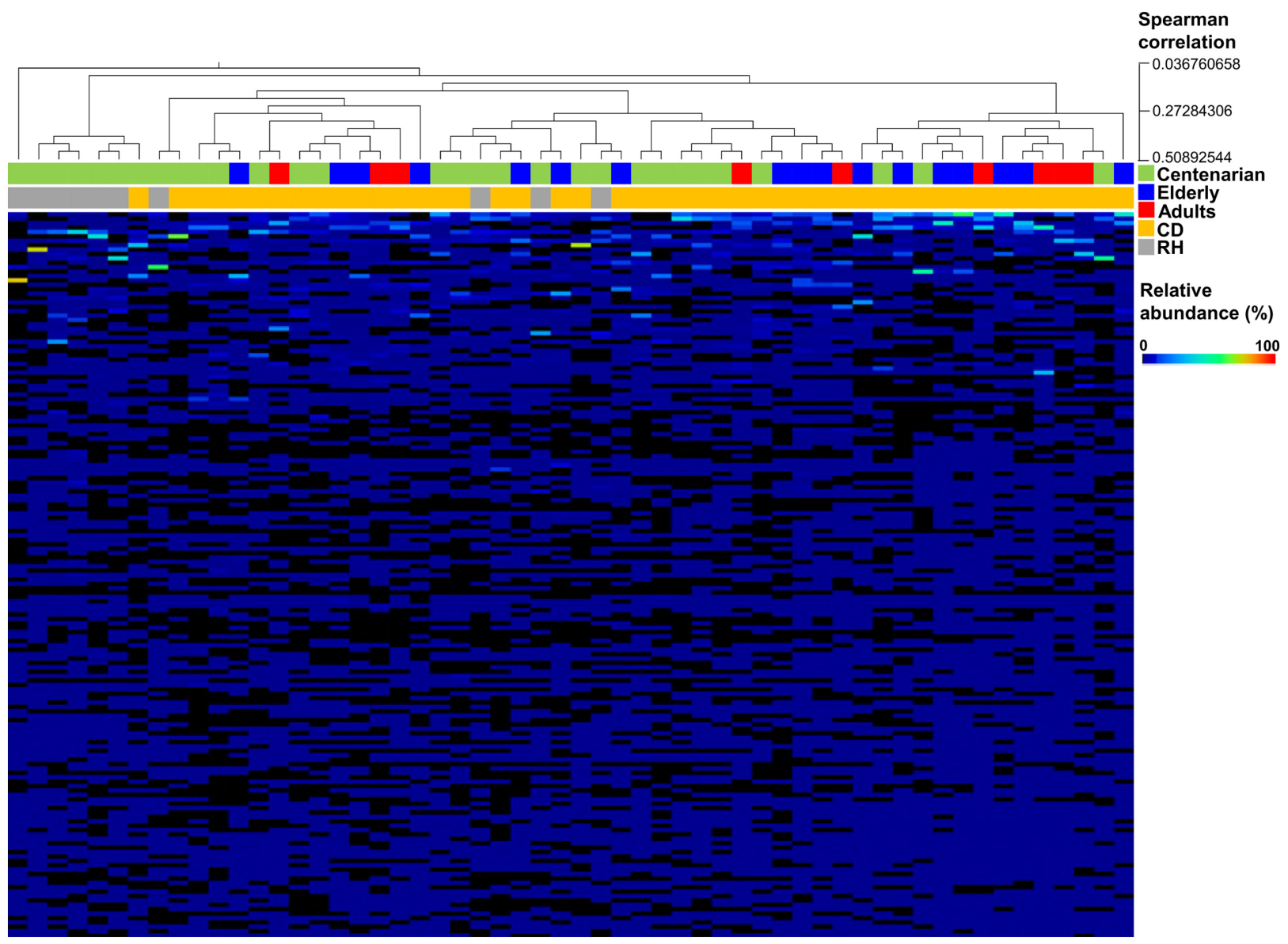

Fig. 3. Heatmap analysis of genera detected in the gut microbiota of subjects in longevity villages.

Clustering analysis was conducted using the Spearman rank correlation. Yellow green indicates centenarians, blue indicates the elderly, red indicates adults, yellow indicates community-dwelling (CD), and gray indicates rehabilitation hospital (RH). 
A
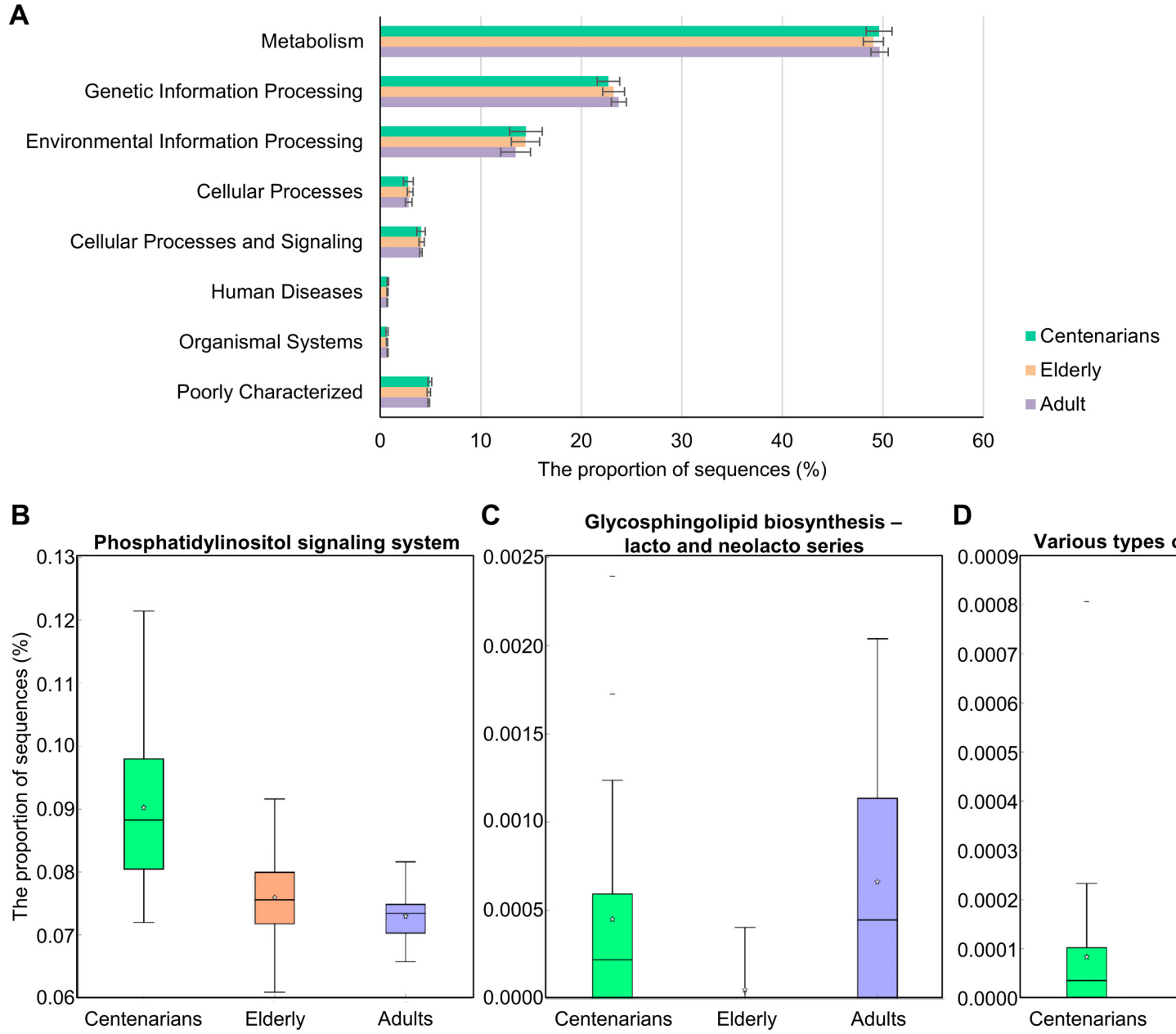

C Glycosphingolipid biosynthesis - D

Fig. 4. Comparison of predicted KEGG pathway between groups.

(A) The highest KEGG categories were compared among groups. (B) The proportions of phosphatidylinositol signaling system among groups (corrected $p<0.05$ ). (C) The comparison of predicted glycosphingolipid biosynthesis from gut microbiome among groups (corrected $p<0.05)$. (D) The comparison of various types of $\mathrm{N}$-glycan biosynthesis among groups (corrected $p<0.05$ ).

regardless of aging (Figs.4B-4D). The proportion of phosphatidylinositol signaling system was higher in the gut microbiomes of centenarians than those in the other two groups. Glycosphingolipid biosynthesis-related microbiota was higher in centenarians and adults than that in the elderly. The proportion of various types of N-glycan biosynthesis was higher in the gut microbiomes of centenarians than those in the elderly and adults.

\section{Comparison of Obtained Centenarian Gut Microbiota between Community-Dwelling and Rehabilitation Hospital Residents}

In the heatmap analysis, the gut microbiota obtained from centenarians in rehabilitation hospitals was relatively clustered together, and separated from community-dwelling centenarians (Fig. 3). The difference in gut microbiota between centenarians from rehabilitation hospitals and community-dwelling subjects was analyzed. The diversity indices (observed OTUs and Shannon diversity) of the community-dwelling centenarians were higher than that of the centenarians in rehabilitation hospitals (Figs. 5A and $5 \mathrm{~B})$. However, the difference of diversity indices was not statistically significant $(p>0.05)$. The average compositions of phyla in bacterial communities also showed differences between centenarians who were community-dwelling and those living in rehabilitation hospitals (Fig. 5C). The proportions of Firmicutes (67.0\%) and Tenericutes (3.0\%) were higher in gut microbiota of community-dwelling subjects than those $(61.2 \%$ and $1.1 \%)$ in rehabilitation hospitals, whereas Bacteroidetes, Proteobacteria, Actinobacteria, and 


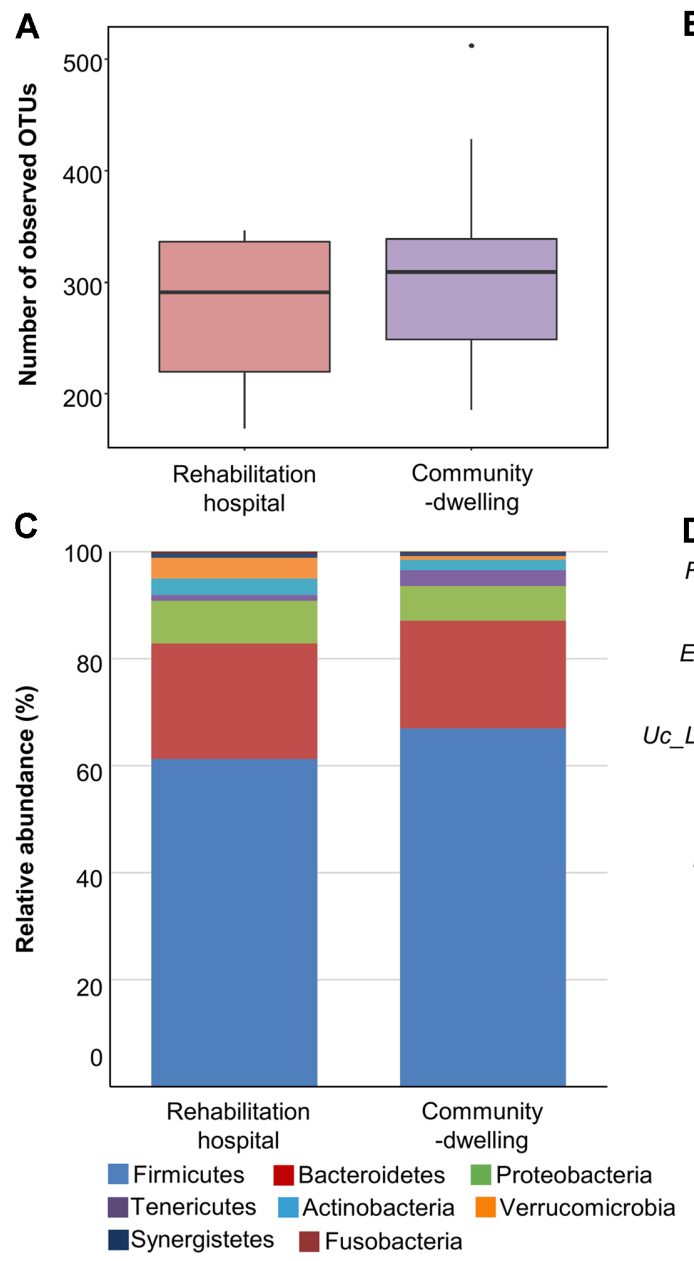

B

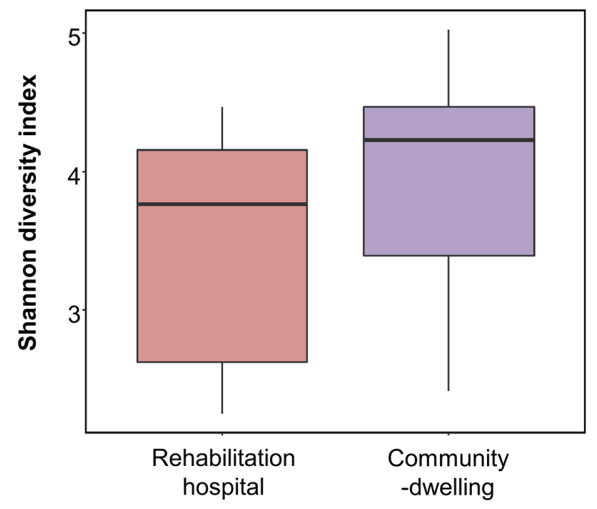

D

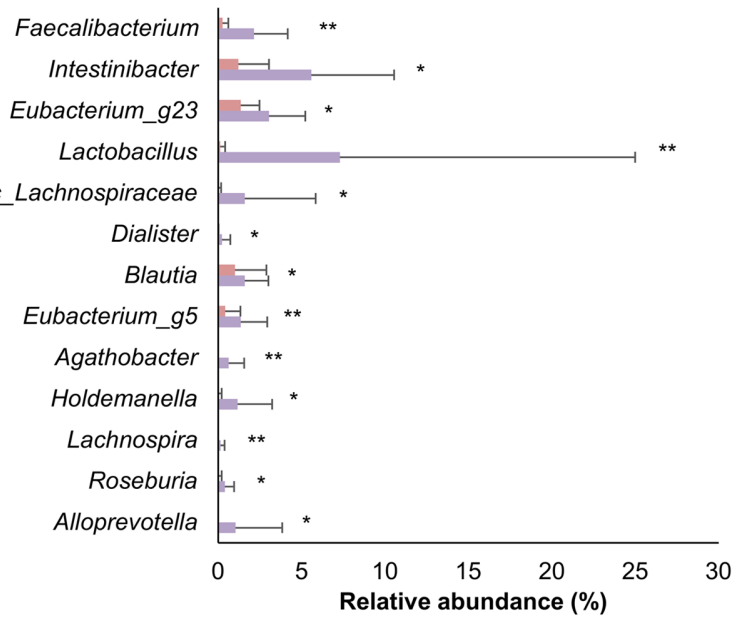

Rehabilitation hospital Community-dwelling

Fig. 5. Comparison of the gut microbiota between centenarians in rehabilitation hospital and community-dwelling groups.

(A) Comparison of observed OTUs between rehabilitation hospital and community-dwelling groups. (B) Comparison of estimated Shannon diversity indices between the groups. (C) The average composition of gut microbiota obtained from two groups. (D) Frequently detected genera were compared between groups. The significance of the differences between groups was tested using the Mann-Whitney test. (** $p<0.01 ; * p<0.05)$.

Verrucomicrobia were higher in the centenarians in rehabilitation hospitals. The significantly different genera between community-dwelling subjects and rehabilitation hospital subjects were detected (Fig. 5D). Detected genera were higher in community-dwelling centenarians than subjects in rehabilitation hospitals. The differences of microbiota functions between these two groups were also analyzed. Two metabolic pathways were selected by KruskalWallis H-test with Benjamini-Hochberg correction $(p<0.1)$ (Table S5). Carbohydrate metabolism was significantly different between the two groups (corrected $p$ value $<0.05$ ).

\section{Discussion}

In this study, the gut microbiota of 30 centenarians in longevity villages in South Korea was analyzed and compared with those of the elderly and adults in the same regions and urbanized towns. The gut microbiota were different between subjects in longevity villages and urbanized towns and the different members of gut microbiota obtained from these two groups were detailed at each taxonomic level. The gut microbiota of centenarians was also different from those of the elderly and adults in the same regions. Furthermore, the predicted functions of gut microbiota can be associated with beneficial effects in centenarians.

The decreased ability to chew, loss of teeth, and loss of gustatory perception could change the habits of diet and appetite of the elderly and the changed diet could affect the gut microbiota $[12,35]$. In this study, the investigation about the dietary characteristics of centenarians living in longevity villages showed that these individuals had the 
dietary habit of eating regular three meals with appetite. Although the frequencies of intake of eggs were lower in the centenarians compared with that in adults, the frequencies of consuming other food groups and proteincontaining food were not different to the elderly and adults in the same regions. These results are in accordance with a previous study that included 91 elderly people over 90 years old residing in the same southwestern longevity belts in Korea [36]. They reported that the characteristic dietary feature of the elderly people over 90 years included having three regular meals a day and taking meals with their family. In addition, most subjects had good appetite and felt the pleasure of eating. These results suggested that the centenarians living in the longevity villages could take sufficient and diverse foods during their meals.

The gut microbiota of subjects in longevity villages was significantly different to those in urbanized towns. Differences of gut microbiota between subjects in longevity villages and urbanized towns were reported in a previous study [16]. They reported that the proportions of Bacteroidetes were higher in subjects of longevity villages than urbanized towns and Firmicutes was lower in longevity villages. However, these results were in contrast to our observations in this study (Fig. 1). This discrepancy could be due to the number of compared subjects, age of subjects, and analysis methods. Most of the downloaded sequences used in the previous study were removed by criteria of read number, age information, and quality filtering step. The number of young adults $(n=2)$ was relatively small, and the centenarians were not included in the previous study. However, 11 young adults in longevity villages and 22 adults in urbanized towns from two different studies were included in this study.

The relative abundances of Intestinibacter, Romboutsia, Turicibacter, and Eubacterium_g5 were higher in subjects of longevity villages than urbanized towns in this study (Fig. S2). Intestinibacter is resistant to oxidative stress and indicative of an indirect involvement in mucus degradation [37]. Romboutsia is a commensal bacteria in the gut and is adapted to a nutrient-rich environment [38]. Turicibacter was implicated in the production of butyrate in response to a fat-rich diet [39]. However, the relative abundances of Lachnospira, Roseburia, and Bacteroides were lower in subjects of longevity villages than those of urbanized towns. Lachnospira and Roseburia can produce butyrate in the colon and are known pectin degraders that play important roles in the colonic fermentation of dietary fibers $[40,41]$. The higher proportion of Bacteroides in gut microbiota is associated with dietary patterns of protein and animal fats and fiber-utilizing bacteria [9, 42]. These bacteria are commensal bacteria in gut environments and the differences observed could be due to the long-term diet patterns between longevity villages and urbanized towns. Gut microbiota can have an influence on host health through the amounts and profiles of short-chain fatty acids (SCFAs) produced from the same carbohydrate substrates [43]. Thus, the different gut microbiota between longevity villages and urbanized towns could have an influence on healthy aging. A total of 146 predicted pathways of gut microbiota were significantly different between subjects of longevity villages and urbanized towns (corrected $p<0.05$; Table S6). This result also shows that the influence of gut microbiota on host health could be different among subjects from longevity villages and urbanized towns.

The composition of gut microbiota in the centenarians showed different characteristics from those of the elderly and the adults even within the same longevity villages. The proportions of Bacteroidetes were higher in centenarians, whereas those of Firmicutes were lower compared to that of the elderly (Fig. 1C). Similar results in the centenarians were reported in previous study [14], the composition of phylum Firmicutes was decreased, while the composition of phylum Bacteroidetes was increased in the centenarians. Bacteroides spp., within Bacteroidetes phylum, are commensal bacteria in the human gut and degrade polysaccharides and isoflavones to absorb energy sources [44, 45]. Isoflavone can be produced by eating soy protein; thus the relatively high abundance of Bacteroides in centenarians could be related with their diet (Fig. S2). The relative abundances of Faecalibacterium and Prevotella were lower in centenarians compared with other groups, and Escherichia within Proteobacteria was increased along with aging (Fig. 2). This result was similar to the age-related rearrangement of gut microbiota in previous studies [12, 14, 15, 17, 18]. With increasing age, a decreased capability of coping with exogenous stressors, increased level of pro-inflammatory markers, and chronic low-grade inflammation were found [46]. The chronic low-grade inflammation in the elderly can be common pathogenic mechanisms of age-related diseases such as metabolic syndrome, type 2 diabetes, and cardiovascular diseases [46, 47]. However, the centenarians had increased anti-inflammatory characteristics with increased level of pro-inflammatory markers, allowing them to counterbalance the chronic low-grade inflammation associated with aging [47, 48]. Good immunological and metabolic health-related bacteria, such as Akkermansia, Christensenellaceae, and Lactobacillus, were higher in centenarians than other groups, similar to previous report 
(Figs. 2 and S2) [18,49]. The relative abundance of Clostridium was higher in centenarians in longevity villages, compared with other groups in longevity villages and urbanized towns, similar to previous studies [50,51]. The commensal Clostridium plays a key role in modulating gut homeostasis during the entire lifespan. The abundance of Collinsella can be associated with dietary patterns [52, 53]. We propose that the centenarians' dietary habits and taking diverse foods from their meals may affect the gut microbiota and promote the abundance of those associated with healthy aging in the centenarians.

Three metabolic pathways of gut microbiota were predicted to be abundant in the centenarians, regardless of aging (Fig. 4). The phosphatidylinositol signaling system can be more highly enriched in centenarians than in the elderly and adults. Previous studies reported that the phosphatidylinositol signaling system is associated with gut inflammation, regulation of immune system, and maintaining energy homeostasis [54, 55]. Dysregulation of the phosphatidylinositol signaling system can promote inflammation and various diseases. Therefore, the higher abundance of predicted phosphatidylinositol signaling system in gut microbiota of centenarians can be associated with anti-inflammation and healthy status of the gut. Glycosphingolipid biosynthesis pathway was predicted to be higher in centenarians and adults than in the elderly. Glycosphingolipid can reduce the invariant NKT cells in the lamina propria and decrease severity of disease in mouse model study [56]. Thus, the higher proportion of glycosphingolipid biosynthesis in centenarians can be associated with healthy function of gut microbiota. Various types of $\mathrm{N}$-glycan biosynthesis were predicted to be higher in the gut microbiota of centenarians than in the elderly and adults. N-glycan biosynthesis can enhance bacterial fitness by protecting bacterial proteins from gut proteases [57]. This pathway can be associated with the stability of gut microbiome in centenarians. Therefore, predicted functions of the gut microbiota of centenarians in this study could be associated with their health compared to those from the elderly and adults.

When comparing the composition of gut microbiota between the centenarians living in the community and rehabilitation hospitals, the proportions of Bacteroidetes and Proteobacteria were higher in rehabilitation hospital subjects and the bacterial diversity was lower compared to the centenarians who were community-dwelling (Fig. 5). The proportion of Faecalibacterium, whose abundance is agerelated, was lower in the centenarians living in rehabilitation hospitals than in the community-dwellers $(p<0.01)$. The dietary habits between community-dwelling centenarians and those in rehabilitation hospitals were detected (Table S7). Previous study also reported that the elderly subjects living in the community consumed diverse healthy food and a concomitant gut microbiota diversity, while the elderly subjects living in rehabilitation hospitals ate less diverse healthy food and had less gut microbiota diversity [12]. Therefore, the gut microbiota of centenarians were influenced by living environment and dietary lifestyle.

There are several limitations to this study. The gut microbiota of centenarians were compared with the elderly and adults subjects with a cross-sectional design at a single time point. The characteristics of gut microbiota of the elderly and adults, which can be healthy aging, could not be identified, as this would necessitate long-term investigations. The information of centenarians' offspring, genetic and environmental and lifestyle determinants of health aging could not be analyzed. In addition, the agematched subjects for the elderly in urbanized towns were relatively small due to the criteria of a sequence quality filtering process in the present study. However, the present study analyzed 30 centenarians in longevity villages, and differences of gut microbiota and predicted functions are provided. This information could contribute to the modulation of gut microbiota for healthy aging. Further studies with a larger sample size, metabolomics, and analyses of host-microbiome interactions with host genetic, immunologic, and metabolic factors are needed to identify the role of gut microbiota in centenarians.

In conclusion, we identified the different members of gut microbiota in centenarians compared with those in the elderly and adults in longevity villages and urbanized towns. The dietary characteristics of the centenarians in longevity villages showed that the centenarians had regular dietary habits with diverse food intake. This can influence the composition of the gut microbiota of centenarians. Different gut microbiota in centenarians were predicted to be associated with the phosphatidylinositol signaling system, glycosphingolipid biosynthesis, and various types of $\mathrm{N}$-glycan biosynthesis. These functions of gut microbiota can be related to the healthy gut environment of centenarians. Further studies are needed to validate the function of the gut microbiota of the centenarians and the influences of diet in longevity villages on the formation of the gut microbiota in centenarians. This study provides information on the gut microbiota of centenarians and helps to identify characteristics of gut microbiota that enable healthy aging in human beings. 


\section{Acknowledgments}

This study was supported by the grant of Korea Health Technology R\&D Project through the Korea Health Industry Development Institute (KHIDI), funded by the Ministry of Health \& Welfare, Republic of Korea (HI14C1277); and Hallym University Research Fund, 2018 (HRF-201808-006).

\section{Conflict of Interest}

The authors have no financial conflicts of interest to declare.

\section{References}

1. Willcox DC, Willcox BJ, He Q, Wang NC, Suzuki M. 2008. They really are that old: a validation study of centenarian prevalence in Okinawa. J. Gerontol. A, Biol. Sci. Med. Sci. 63: 338-349.

2. Smith DW. 1997. Centenarians: human longevity outliers. Gerontologist 37: 200-206.

3. Bernstein AM, Willcox BJ, Tamaki H, Kunishima N, Suzuki M, Willcox DC, et al. 2004. First autopsy study of an Okinawan centenarian: absence of many age-related diseases. $J$. Gerontol. A, Biol. Sci. Med. Sci. 59: 1195-1199.

4. Evert J, Lawler E, Bogan H, Perls T. 2003. Morbidity profiles of centenarians: survivors, delayers, and escapers. J. Gerontol. A, Biol. Sci. Med. Sci. 58: 232-237.

5. Franceschi C, Passarino G, Mari D, Monti D. 2017. Centenarians as a 21st century healthy aging model: A legacy of humanity and the need for a world-wide consortium (WWC100+). Mech. Ageing Dev. 165: 55-58.

6. Kheirbek RE, Fokar A, Shara N, Bell-Wilson LK, Moore HJ, Olsen E, et al. 2017. Characteristics and incidence of chronic illness in community-dwelling predominantly male U.S. Veteran centenarians. J. Am. Geriatr. Soc. 65: 2100-2106.

7. Perls T, Levenson R, Regan M, Puca A. 2002. What does it take to live to 100? Mech. Ageing Dev. 123: 231-242.

8. Yatsunenko T, Rey FE, Manary MJ, Trehan I, DominguezBello MG, Contreras M, et al. 2012. Human gut microbiome viewed across age and geography. Nature 486: 222-227.

9. $\mathrm{Wu}$ GD, Chen J, Hoffmann C, Bittinger $\mathrm{K}$, Chen YY, Keilbaugh SA, et al. 2011. Linking long-term dietary patterns with gut microbial enterotypes. Science 334: 105-108.

10. Zhang H, DiBaise JK, Zuccolo A, Kudrna D, Braidotti M, Yu Y, et al. 2009. Human gut microbiota in obesity and after gastric bypass. Proc. Natl. Acad. Sci. USA 106: 2365-2370.

11. $\mathrm{Wu}$ GD, Lewis JD. 2013. Analysis of the human gut microbiome and association with disease. Clin. Gastroenterol. Hepatol. 11: 774-777.

12. Claesson MJ, Jeffery IB, Conde S, Power SE, O'Connor EM, Cusack S, et al. 2012. Gut microbiota composition correlates with diet and health in the elderly. Nature 488: 178-184.
13. Candela M, Biagi E, Brigidi P, O'Toole PW, De Vos WM 2014. Maintenance of a healthy trajectory of the intestinal microbiome during aging: a dietary approach. Mech. Ageing Dev. 136-137: 70-75.

14. Biagi E, Nylund L, Candela M, Ostan R, Bucci L, Pini E, et al. 2010. Through ageing, and beyond: gut microbiota and inflammatory status in seniors and centenarians. PLoS One 5: e106967.

15. Wang F, Yu T, Huang G, Cai D, Liang X, Su H, et al. 2015. Gut microbiota community and its assembly associated with age and diet in Chinese centenarians. J. Microbiol. Biotechnol. 25: 1195-1204.

16. Park SH, Kim KA, Ahn YT, Jeong JJ, Huh CS, Kim DH. 2015. Comparative analysis of gut microbiota in elderly people of urbanized towns and longevity villages. BMC Microbiol. 15: 49.

17. Odamaki T, Kato K, Sugahara H, Hashikura N, Takahashi S, Xiao JZ, et al. 2016. Age-related changes in gut microbiota composition from newborn to centenarian: a cross-sectional study. BMC Microbiol. 16: 90.

18. Biagi E, Franceschi C, Rampelli S, Severgnini M, Ostan R, Turroni S, et al. 2016. Gut microbiota and extreme longevity. Curr. Biol. 26: 1480-1485.

19. Guigoz Y, Dore J, Schiffrin EJ. 2008. The inflammatory status of old age can be nurtured from the intestinal environment. Curr. Opin. Clin. Nutr. Metab. Care 11: 13-20.

20. Woodmansey EJ. 2007. Intestinal bacteria and ageing. J. Appl. Microbiol. 102: 1178-1186.

21. Buford TW. 2017. (Dis)Trust your gut: the gut microbiome in age-related inflammation, health, and disease. Microbiome 5: 80.

22. Santoro A, Ostan R, Candela M, Biagi E, Brigidi P, Capri M, et al. 2018. Gut microbiota changes in the extreme decades of human life: a focus on centenarians. Cell Mol. Life Sci. 75: 129-148.

23. Hur M, Kim Y, Song HR, Kim JM, Choi YI, Yi H. 2011. Effect of genetically modified poplars on soil microbial communities during the phytoremediation of waste mine tailings. Appl. Environ. Microbiol. 77: 7611-7619.

24. Jeon YS, Chun J, Kim BS. 2013. Identification of household bacterial community and analysis of species shared with human microbiome. Curr. Microbiol. 67: 557-563.

25. Kim BS, Bae HS, Lim CY, Kim MJ, Seo JG, Kim JY, et al. 2013. Comparison of gut microbiota between sasang constitutions. Evid. Based Complement. Alternat. Med. 2013: 171643.

26. Edgar RC. 2010. Search and clustering orders of magnitude faster than BLAST. Bioinformatics 26: 2460-2461.

27. Edgar RC. 2013. UPARSE: highly accurate OTU sequences from microbial amplicon reads. Nat. Methods 10: 996-998.

28. Werner JJ, Koren O, Hugenholtz P, DeSantis TZ, Walters WA, Caporaso JG, et al. 2012. Impact of training sets on classification of high-throughput bacterial 16S rRNA gene surveys. ISME J. 6: 94-103.

29. Yoon SH, Ha SM, Kwon S, Lim J, Kim Y, Seo H, et al. 2017. Introducing EzBioCloud: a taxonomically united database of 
16S rRNA gene sequences and whole-genome assemblies. Int. J. Syst. Evol. Microbiol. 67: 1613-1617.

30. Schloss PD, Westcott SL, Ryabin T, Hall JR, Hartmann M, Hollister EB, et al. 2009. Introducing mothur: open-source, platform-independent, community-supported software for describing and comparing microbial communities. Appl. Environ. Microbiol. 75: 7537-7541.

31. Zakrzewski M, Proietti C, Ellis JJ, Hasan S, Brion MJ, Berger B, et al. 2017. Calypso: a user-friendly web-server for mining and visualizing microbiome-environment interactions. Bioinformatics 33: 782-783.

32. Segata N, Izard J, Waldron L, Gevers D, Miropolsky L, Garrett WS, et al. 2011. Metagenomic biomarker discovery and explanation. Genome Biol. 12: R60.

33. Langille MG, Zaneveld J, Caporaso JG, McDonald D, Knights D, Reyes JA, et al. 2013. Predictive functional profiling of microbial communities using 16S rRNA marker gene sequences. Nat. Biotechnol. 31: 814-821.

34. Bluman AG. 2007. Elementary statistics: A step by step approach, 6th Ed. McGraw Hill Higher Education, New York.

35. Jeffery IB, O'Toole PW. 2013. Diet-microbiota interactions and their implications for healthy living. Nutrients 5: 234-252.

36. Lee M. 2005. The dietary habits of the nonagenarian population in longevity belt in Korea. Korean J. Community Nutr. 10: 513-524.

37. Forslund K, Hildebrand F, Nielsen T, Falony G, Le Chatelier E, Sunagawa $S$, et al. 2015. Disentangling type 2 diabetes and metformin treatment signatures in the human gut microbiota. Nature 528: 262-266.

38. Gerritsen J, Hornung B, Renckens B, van Hijum S, Martins Dos Santos VAP, Rijkers GT, et al. 2017. Genomic and functional analysis of Romboutsia ilealis CRIB(T) reveals adaptation to the small intestine. PeerJ. 5: e3698.

39. Zhong Y, Nyman M, Fak F. 2015. Modulation of gut microbiota in rats fed high-fat diets by processing wholegrain barley to barley malt. Mol. Nutr. Food Res. 59: 2066-2076.

40. Rode LM, Genthner BR, Bryant MP. 1981. Syntrophic association by cocultures of the methanol- and $\mathrm{CO}_{2}-\mathrm{H}_{2}-$ utilizing species Eubacterium limosum and pectin-fermenting Lachnospira multiparus during growth in a pectin medium. Appl. Environ. Microbiol. 42: 20-22.

41. Marounek M, Duskova D. 1999. Metabolism of pectin in rumen bacteria Butyrivibrio fibrisolvens and Prevotella ruminicola. Lett. Appl. Microbiol. 29: 429-433.

42. Lim MY, Rho M, Song YM, Lee K, Sung J, Ko G. 2014. Stability of gut enterotypes in Korean monozygotic twins and their association with biomarkers and diet. Sci. Rep. 4: 7348.

43. Chen T, Long W, Zhang C, Liu S, Zhao L, Hamaker BR. 2017. Fiber-utilizing capacity varies in Prevotella- versus Bacteroides-dominated gut microbiota. Sci. Rep. 7: 2594.
44. Hobbs ME, Williams HJ, Hillerich B, Almo SC, Raushel FM. 2014. 1-Galactose metabolism in Bacteroides vulgatus from the human gut microbiota. Biochemistry 53: 4661-4670.

45. Renouf M, Hendrich S. 2011. Bacteroides uniformis is a putative bacterial species associated with the degradation of the isoflavone genistein in human feces. J. Nutr. 141: 1120-1126.

46. Cevenini E, Monti D, Franceschi C. 2013. Inflamm-ageing. Curr. Opin. Clin. Nutr. Metab. Care 16: 14-20.

47. De Martinis M, Franceschi C, Monti D, Ginaldi L. 2005. Inflamm-ageing and lifelong antigenic load as major determinants of ageing rate and longevity. FEBS Lett. 579: 2035-2039.

48. Franceschi C, Capri M, Monti D, Giunta S, Olivieri F, Sevini F, et al. 2007. Inflammaging and anti-inflammaging: a systemic perspective on aging and longevity emerged from studies in humans. Mech. Ageing Dev. 128: 92-105.

49. Jeong JJ, Kim KA, Hwang YJ, Han MJ, Kim DH. 2016. Antiinflammaging effects of Lactobacillus brevis OW38 in aged mice. Benef. Microbes 7: 707-718.

50. Drago L, Toscano M, Rodighiero V, De Vecchi E, Mogna G. 2012. Cultivable and pyrosequenced fecal microflora in centenarians and young subjects. J. Clin. Gastroenterol. 46 (Suppl): S81-84.

51. Lopetuso LR, Scaldaferri F, Petito V, Gasbarrini A. 2013. Commensal Clostridia: leading players in the maintenance of gut homeostasis. Gut Pathog. 5: 23.

52. Gomez-Arango LF, Barrett HL, Wilkinson SA, Callaway LK, McIntyre HD, Morrison M, et al. 2017. Low dietary fiber intake increases Collinsella abundance in the gut microbiota of overweight and obese pregnant women. Gut Microbes 9: 189-201.

53. Angelakis E, Yasir M, Bachar D, Azhar EI, Lagier JC, Bibi F, et al. 2016. Gut microbiome and dietary patterns in different Saudi populations and monkeys. Sci. Rep. 6: 32191.

54. Thakur PC, Davison JM, Stuckenholz C, Lu L, Bahary N. 2014. Dysregulated phosphatidylinositol signaling promotes endoplasmic-reticulum-stress-mediated intestinal mucosal injury and inflammation in zebrafish. Dis. Model Mech. 7: 93-106.

55. Bourassa MW, Alim I, Bultman SJ, Ratan RR. 2016. Butyrate, neuroepigenetics and the gut microbiome: Can a high fiber diet improve brain health? Neurosci. Lett. 625: 56-63.

56. An D, Oh SF, Olszak T, Neves JF, Avci FY, Erturk-Hasdemir D, et al. 2014. Sphingolipids from a symbiotic microbe regulate homeostasis of host intestinal natural killer T cells. Cell 156: 123-133.

57. Alemka A, Nothaft H, Zheng J, Szymanski CM. 2013. Nglycosylation of Campylobacter jejuni surface proteins promotes bacterial fitness. Infect. Immun. 81: 1674-1682. 